\title{
Überaktive Blase: Aufklärungskampagne geht weiter
}

_ In Deutschland sind Millionen Frauen wie Männer von der Überaktiven Blase betroffen: Bei den 50-Jährigen ist dies statistisch gesehen jeder fünfte. Aber auch jüngere Menschen kann es treffen. „Unser Ziel ist es, zum einen möglichst viele bislang still vor sich hin leidende Betroffene zu motivieren, sich an ihren Arzt zu wenden, um so den Weg zu Diagnose und nachhaltig wirksamer Therapie zu ebnen", so Dr. Thilo Pfeuffer, Leiter Produktmanagement Urologie bei Astellas Pharma. „Zum anderen müssen wir aber auch die Wahrnehmung erhöhen, dass die Überaktive Blase eine behandelbare Erkrankung ist und keine hinzunehmende Gesundheitsstörung." Seit Oktober 2011 führt das Unternehmen seine erfolgreiche Aufklärungskampagne zum Tabu-The- ma "Überaktive Blase“, die im Frühjahr 2010 begann, daher fort. An der Kampagne sind neben Astellas auch Arztpraxen und Apotheken beteiligt, denen das Unternehmen kostenlos Informationsmaterial sowie Patientenbroschüren zur Verfügung stellt.

Unter dem Motto "Sie bestimmen Ihr Leben. Nicht Ihre Blase" klärt die Internetseite www.blase-ok.de Ärzte und Patienten bereits seit Mitte Februar 2011 erfolgreich über das Thema Überaktive Blase auf. Interessierte Praxen, Apotheker und Betroffene können das Informationsmaterial kostenfrei beim Astellas Pharma Kundenservice unter der Telefonnummer 08001114544 anfordern.

Nach Informationen von Astellas, München
Neues zum Harnblasenkarzinom

_ Zum fünften Mal fand im Dezember 2011 das urologische Symposium von medac in Hamburg statt. Geboten wurde hochkarätige Fortbildung auf wissenschaftlichem und berufspolitischem Gebiet unter Leitung von Prof. Jürgen E. Gschwend, München. Wissenschaftliches Schwerpunktthema war das Harnblasenkarzinom. Die Vorträge brachten Neues aus Diagnostik, leitliniengerechtem Vorgehen beim nicht muskelinvasiven Blasenkrebs (NMIBC), zum Stellenwert der funktionserhaltenden Zystektomie beim Hochrisiko-NMIBC sowie zur systemischen Therapie. Auch die Berufspolitik kam nicht zu kurz. Eher enttäuscht als erfreut zeigte sich der niedergelassene Urologe Dr. Wolfgang Rulf, Erkrath: Eine Alternative zur Resignation sei aber die Eigeninitiative, zum Beispiel durch Gründung von Berufsausübungsgemeinschaften.

Nach Informationen von medac, Wedel

\section{Neuer Online-Service: 100 Erreger auf einen Klick}

- Der Online-Service des BODE SCIENCE CENTER (www.bode-science-center.de) hat kompakt und praxisnah Informationen über rund 100 klinisch relevante Krankheitserreger zusammengestellt. User finden auf einen Blick alle wichtigen Informationen zu Familie, Vorkommen, den häufigsten Infektionen durch den Erreger sowie zum Resistenzverhalten. Als besonderer Service für die Umsetzung des Hygiene- oder Ausbruchsmanagements in Einrichtungen, wird bereits in der A-Z Übersicht zu jedem Erreger das erforderliche Wirkungsspektrum für ein Desinfektionsverfahren angezeigt. Eine praxisnahe Unterstützung für Hygieneverantwortliche, um mit den richtigen Desinfektionsmaßnahmen schnell auf Krankheitserreger reagieren zu können.

Der Online-Service berücksichtigt auch Erreger abseits der "üblichen Verdächtigen", den Staphylokokken und Enterokokken. In die Liste aufgenommen wurden unter anderem die seltener auftretenden, aber als Nosokomialkeime gefürchteten Spezies Burkholderia cepacia, Acinetobacter Iwoffii und Bacteroides fragilis. Als zusätzlicher Service werden in Pop-up-Fenstern die Kategorien Bakterizidie, Fungizidie, Levurozidie, Mykobakterizidie,
Tuberkulozidie, Sporizidie, begrenzte Viruzidie und Viruzidie verständlich erklärt. Die Online-Erregersuche ist nur ein Bestandteil eines umfassenden Services, den das neue BODE SCIENCE CENTER interessierten Kreisen bietet. Das unter dem Dach der HARTMANN AG, Heidenheim, neu gegründete Kompetenzzentrum verbindet Wissenschaft und Praxis im Infektionsschutz. Zu den Kernleistungen gehören eigene sowie Reviews von Publikationen zu grundlegenden Themen, die Entwicklung neuer Standards und eine fachliche Beratung. Auf der Website stehen zum Beispiel aktuelle Studien-Abstracts zu nosokomialen Infektionen, zu Infektionsschutzmaßnahmen, neuen Standards zum Download zur Verfügung. Ergänzt wird die wissenschaftliche Aufbereitung zentraler Infektions- schutzthemen durch praktische Anleitungen für ein optimiertes Hygienemanagement, darunter Poster oder Schulungsfilme ebenfalls zum Download.

Nach Informationen von

Hartmann, Heidenheim

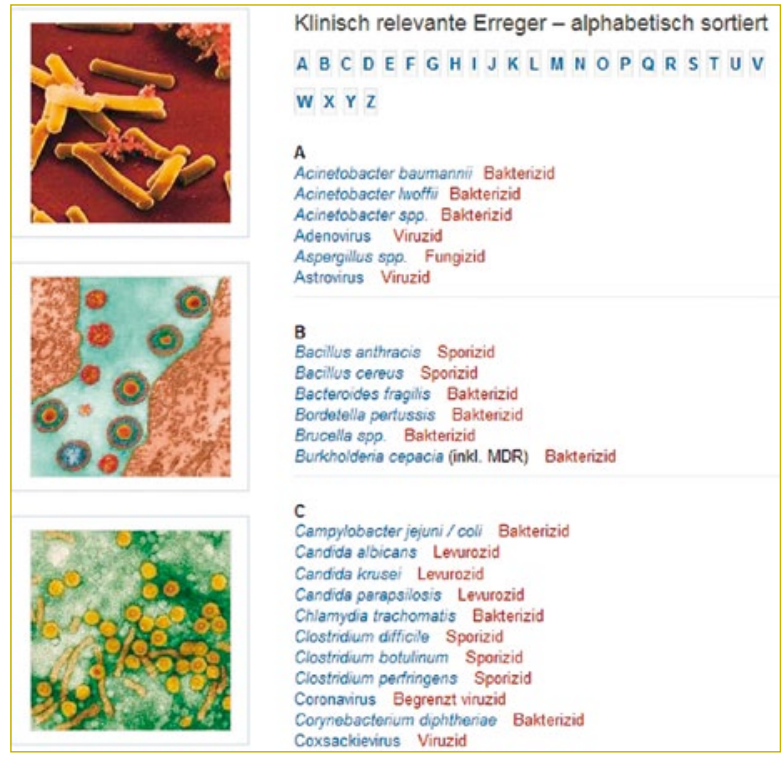

Erregersuche leicht gemacht 